\title{
Immunophenotype identifies children with immune cytopenias at risk of inborn errors of immunity-related mutations
}

\author{
Daniele Zama ${ }^{1}$, Francesca Conti $^{1}$, Mattia Moratti ${ }^{2}$, Maria Cantarini ${ }^{1}$, Elena Facchini ${ }^{1}$, \\ Beatrice Rivalta ${ }^{1}$, Roberto Rondelli ${ }^{3}$, Arcangelo Prete ${ }^{1}$, Simona Ferrari ${ }^{1}$, Marco Seri $^{1}$, and \\ Andrea Pession ${ }^{3}$ \\ ${ }^{1}$ Azienda Ospedaliero-Universitaria di Bologna Policlinico Sant'Orsola-Malpighi \\ ${ }^{2}$ University Hospital of Bologna Sant'Orsola-Malpighi Polyclinic \\ ${ }^{3}$ University of Bologna
}

November 3, 2020

\begin{abstract}
Background Immune thrombocytopenia (ITP), autoimmune hemolytic anemia (AIHA) and autoimmune neutropenia (AIN) are disorders characterized by immune-mediated destruction of hematopoietic cell lineages. A link between pediatric immune cytopenias and inborn errors of immunity (IEI) was established in particular in the combined and chronic forms. Objective Identification of predictive factors of IEI in immune cytopenic children is an important objective for a prompt immunological diagnosis and appropriate management. Aim of this study is to detect clinical and laboratory signs of IEI, in particular the latter by an extensive lymphocyte immunophenotyping. Methods We retrospectively collected 47 pediatric patients with at least one hematological disorder among which persistent/chronic ITP, AIHA and AIN, aged 0-18 years at onset of immune cytopenias and/or immune-dysregulation. The cohort was divided into 2 groups (IEI + and IEI-), based on the presence/absence of underlying IEI diagnosis. IEI+ group, formed by 19/47 individuals, included: Common variable immune deficiency (9/19), Autoimmune lymphoproliferative syndrome (4/19), DiGeorge syndrome (1/19) and unclassified IEI (5/19). Results IEI prevalence among patients with ITP, AIHA, AIN and Evans Syndrome was respectively of $42 \%, 64 \%, 36 \%$ and $62 \%$. In IEI+ the extended lymphocyte immunophenotyping identified the presence of statistically significant ( $\mathrm{p}$-value $<0.05)$ specific characteristics, namely T/B lymphopenia, decrease in naïve T-cells\%, switched memory B-cells\%, plasmablasts\% and/or immunoglobulins, increase in effector/central memory T-cells\% and CD21low B-cells\%. Conclusion A wide focused clinical/immunophenotypical characterization of pediatric patients with immune cytopenia can highlight specific signs of IEI, potentially helpful in the diagnostic and clinical management, identifying children worthy of IEI-related molecular analysis.
\end{abstract}

Immunophenotype identifies children with immune cytopenias at risk of inborn errors of immunity-related mutations

Daniele Zama* ${ }^{*}$ PhD; Francesca Conti ${ }^{*}$, PhD; Mattia Moratti, MD; Maria E. Cantarini, MD; Elena Facchini, MD; Beatrice Rivalta, MD; Roberto Rondelli, MD; Arcangelo Prete, MD; Simona Ferrari, MD; Marco Seri, Prof.; Andrea Pession, Prof.

*These authors contributed equally to the manuscript.

Affiliation Department of Pediatrics, Azienda Ospedaliero - Universitaria Policlinico di Sant'Orsola, Bologna, Italy

Running title Immune deficiency in immune cytopenias

Address correspondence to Daniele Zama, PhD, Pediatric Oncology and Hematology Unit "Lalla Seràgnoli, Azienda Ospedaliero - Universitaria Policlinico di Sant'Orsola, Via Massarenti 11, 40137 Bologna, 
Italy;daniele.zama@aosp.bo.it; +390516364490; Fax +390516363400.

Word count manuscript 2500

Word count abstract 250

Number of tables 4

Number of figures 2

Material in the electronic repository Supplementary table

Conflicts of Interest All patients and legal tutors provided written informed consent to participate in this study. Ethics Committee of Bologna University-Hospital Sant'Orsola approved the study protocols. The authors have no financial relationships and/or competing interests relevant to this article to disclose.

Financial Support No funding was secured for this study.

\section{Abstract}

Background Immune thrombocytopenia (ITP), autoimmune hemolytic anemia (AIHA) and autoimmune neutropenia (AIN) are disorders characterized by immune-mediated destruction of hematopoietic cell lineages. A link between pediatric immune cytopenias and inborn errors of immunity (IEI) was established in particular in the combined and chronic forms.

Objective Identification of predictive factors of IEI in immune cytopenic children is an important objective for a prompt immunological diagnosis and appropriate management. Aim of this study is to detect clinical and laboratory signs of IEI, in particular the latter by an extensive lymphocyte immunophenotyping.

Methods We retrospectively collected 47 pediatric patients with at least one hematological disorder among which persistent/chronic ITP, AIHA and AIN, aged 0-18 years at onset of immune cytopenias and/or immune-dysregulation. The cohort was divided into 2 groups (IEI+ and IEI-), based on the presence/absence of underlying IEI diagnosis. IEI+ group, formed by 19/47 individuals, included: Common variable immune deficiency (9/19), Autoimmune lymphoproliferative syndrome (4/19), DiGeorge syndrome (1/19) and unclassified IEI (5/19).

Results IEI prevalence among patients with ITP, AIHA, AIN and Evans Syndrome was respectively of 42\%, $64 \%, 36 \%$ and $62 \%$.

In IEI+ the extended lymphocyte immunophenotyping identified the presence of statistically significant (p-value $<0.05)$ specific characteristics, namely $\mathrm{T} / \mathrm{B}$ lymphopenia, decrease in naïve T-cells\%, switched memory B-cells\%, plasmablasts\% and/or immunoglobulins, increase in effector/central memory T-cells\% and CD21low B-cells\%.

Conclusion A wide focused clinical/immunophenotypical characterization of pediatric patients with immune cytopenia can highlight specific signs of IEI, potentially helpful in the diagnostic and clinical management, identifying children worthy of IEI-related molecular analysis.

Keywords Immune cytopenias; Inborn errors of immunity; Immune thrombocytopenia; Autoimmune hemolytic anemia; Autoimmune neutropenia; Common variable immune deficiency; Autoimmune lymphoproliferative syndrome, DiGeorge syndrome.

\section{Key Message}

This study highlights the importance of an extended lymphocyte typization in pediatric patients with immune cytopenias, defining correlations useful in identifying patients worthy of IEI-related molecular analysis.

\section{INTRODUCTION}

Immune cytopenias are disorders characterized by immune-mediated destruction of hematopoietic cell lineages. The most common form in children is immune thrombocytopenia (ITP), followed by autoimmune 
hemolytic anemia (AIHA) and autoimmune neutropenia (AIN) ${ }^{1}$. In children affected by AIHA, a link between immune cytopenias and IEI was observed in $8-10 \%$ of cases $^{2}$. In a pediatric cohort with Evans syndrome (ES), defined as synchronous or metachronous association between AIHA and ITP, immune-dysregulation phenomena, including IEI, were found in $70 \%$ of the patients ${ }^{3,4}$.

IEI are congenital disorders caused by defects in genes involved in the development, function and regulation of the immune system, resulting not only in increased susceptibility to infections, but also in inflammatory, autoimmune, allergic, non-malignant lymphoproliferative and neoplastic manifestations ${ }^{5-7}$. IEI most frequently associated with immune cytopenias are: Common variable immune deficiency (CVID), Autoimmune lymphoproliferative syndrome (ALPS), Wiskott-Aldrich syndrome and Combined immunodeficiency (CID) ${ }^{5}$. Depending on the different forms of IEI, the pathogenic mechanisms of cytopenias identified thus far are various: humoral and cell-mediated adaptive immunity ${ }^{8}$, immune-dysregulation in form of hemophagocytosis and splenic sequestration secondary to abnormal lymphoproliferation ${ }^{6,9}$, myelodysplasia ${ }^{10}$, primary bone marrow failure and myelosuppression secondary to infections, malignancies and bone marrow lymphocytic infiltration ${ }^{5}$. IEI-related immune cytopenias differ from idiopathic forms on these issues: late onset, multi-lineage involvement, chronic/relapsing course and tendency towards treatment refractoriness ${ }^{1}$. Aim of this study was to assess prevalence and clinical/immunological predictive factors of IEI in children with immune cytopenias, identifying patients worthy of IEI-related molecular analysis.

\section{METHODS}

Design

This retrospective study included 47 patients since 2000 to 2019 suffering from immune cytopenias at Pediatric Hematology and Immunology Units of the University-Hospital Sant'Orsola - Bologna.

Inclusion criteria were:

Diagnosis of at least one hematological disorder among persistent/chronic ITP, AIHA, AIN;

Age 0-18 years at initial presentation of hematological disease and/or of immune-dysregulation.

Persistent and chronic ITP were defined as a platelet count of $<100 \times 109 / \mathrm{L}$ with no known cause lasting respectively for more than 3 and 12 months.

AIHA was defined by anemia (hemoglobin $<-2$ standard deviations) and a positive direct antiglobulin test associated to signs of hemolysis.

AIN was defined by a neutrophil count $<1.5 \times 109 / \mathrm{L}$ after the first year of life and the detection of antineutrophils antibodies using indirect flow cytometry.

ES was defined by the synchronous/metachronous presence of at least two immune cytopenias ${ }^{4}$.

The diagnosis of IEI met the 2019 revised criteria established by the European Society of Immunodeficiencies $(\mathrm{ESID})^{11}$.

We performed an extended clinical-laboratory characterization of the cohort reported in Supplementary table.

Laboratory investigations

Extended lymphocyte immunophenotyping: CD3+CD4+, CD4+CD45RA+CCR7+ (naïve), CD4+CD45RA-CCR7- (effector memory), CD4+CD45RA-CCR7+ (central memory), CD3+CD8+, CD8+CD45RA+CCR7+ (naïve), CD8+CD45RA-CCR7- (effector memory), CD8+CD45RA-CCR7+ (central memory) and CD4 $+\mathrm{CD} 127-\mathrm{CCR} 7+\mathrm{CD} 25++$ (regulatory) T-cells (T-reg), CD19+ (PAN$\mathrm{B}), \mathrm{CD} 19+\mathrm{IgD}+\mathrm{CD} 27+$ (memory), $\mathrm{CD} 19+\operatorname{IgD}-\mathrm{CD} 27+$ (switched memory), $\mathrm{CD} 19+\operatorname{IgM}++\mathrm{CD} 38++$ (transitional) and CD19+CD21+lCD38- (CD21low) B-cells, CD19+IgM- + CD38 ++ (plasmablasts) through multiparametric flow cytometry; 
Immunoglobulin levels (IgG, $\operatorname{IgA}$, IgM) through turbidimetric method.

Immunophenotypical data were age-referenced and gathered at least 6 months after the end of rituximab and mycophenolate mofetile (MMF), and at least 4 months after the end of steroid therapy, except for three patients whose evaluation was executed one day after stopping and/or during a cycle of steroids. Immunoglobulin levels were detected at least 6 months after the end of rituximab and MMF, and at least 2 months after the end of IVIG and steroid therapy, except for two subjects, whose measurement was made respectively during cyclic subcutaneous IgG replacement and MMF therapy.

Molecular analysis

Targeted gene panel design

Diagnostic gene panel was developed on the Ion Torrent platform (Thermo Fisher Scientific).

The custom panel includes 46 genes involved in the pathogenesis of agammaglobulinemia, CVID and IEI immune-dysregulation disorders (Table 1-A/1-B) based on the last update of the ESID classification ${ }^{11,12}$.

The amplicon design was expected to cover $99.97 \%$ of the $182,523 \mathrm{~kb}$ targeted exons with 942 amplicons. For each exon a six bp of padding was included.

Ion Torrent library preparation and sequencing

Ten nanograms of genomic DNA extracted from peripheral blood were used for library preparation. DNA was amplified using the two gene panel primer pools. PCR pools for each sample were combined and indexed using the Ion Xpress Barcode Adapters kit. The amplified libraries were quantified using the qPCR through the Ion Library TaqMan Quantitation Kit.

All samples were diluted at $10 \mathrm{pM}$, then amplicon libraries were pooled for emulsion PCR and chip loading using the Ion Chef system, according to manufacturer's instructions. The final pool of samples was sequenced with Ion GeneStudio S5 system using Ion 510 or Ion 520 Chips.

Ion Torrent Bioinformatic analysis

Sequencing reads were aligned to hg19 reference genome. Variant calling was performed using Ion Torrent suite software v5.12 or Ion Reporter software v5.10. Called variants were filtered by allele frequencies, selecting variants with MAF < $1 \%$ annotated with GnomAD database (https://gnomad.broadinstitute.org/). Nonsense, frameshift and canonical splicing variants were considered potentially pathogenic, while the other variants were classified according to Varsome database (https://varsome.com/). Called variants with the minimum coverage of $20 \mathrm{X}$ were analysed by Integrative Genome Viewer and confirmed by sanger sequencing. All regions covered $<20 \mathrm{X}$ were reanalysed by sanger sequencing.

DiGeorge syndrome screening

The 22q11.21 deletion was tested in 1/47 individuals according to clinical suspicion using the SALSA MLPA probemix P250 (MRC-Holland) following the manufacturer's instructions.

Statistical analysis

Descriptive statistics included means (95\% Confidence Interval) as appropriate for continuous variables and frequency for categorical variables. All analyses were performed with SPSS software version 21 and Microsoft Excel version 2013. Data elaboration, based on the comparison between two groups (IEI+ and IEI-) differing in the presence/absence of a previously diagnosed IEI, aimed to:

Assess statistical significance (p-value $<0.05$ ) of a difference between the groups, through chi-squared tests for frequency and Student t-test for mean.

Describe the correlation $(\mathrm{p}$-value $<0.05)$ between variables within a group, through Pearson correlation coefficient. 


\section{RESULTS}

Hematological presentation

Forty-seven patients were included in the study. ITP was diagnosed in $38 / 47$ subjects (81\%), AIHA in $11 / 47(23 \%)$ and AIN in $11 / 47(23 \%)$. ES affected 10/47 individuals (21\%), presenting with the following associations: ITP and AIHA in 5/10 cases (50\%), ITP and AIN in 2/10 (20\%), ITP, AIHA and AIN in 3/10 (30\%). IEI prevalence among patients with ITP, AIHA, AIN and ES was respectively of $42 \%, 64 \%, 36 \%$ and $60 \%$ (Fig. 1). IEI+ group counted 19 individuals (40\%), affected by CVID (9/19, 47\%), ALPS (4/19, $21 \%)$, DiGeorge syndrome (DGS) $(1 / 19,5 \%)$ and unclassified IEI $(5 / 19,26 \%)$. IEI- group consisted of 28 subjects (60\%). Mean age at initial presentation with immune cytopenias was not significantly higher in IEI+ group (9.7 versus vs 7.9 years, range $0.7-28.5$ years; $\mathrm{p}=0.348$ ) and their mean follow-up was superior in this group (11.3 vs 4.0 years, $0.2-24.9$ years; $\mathrm{p}<0.001$ ), probably due to a more complex clinical course of IEI-related forms. Mean age at time of IEI diagnosis was 15.2 years (6.9-35.1 years) and mean temporal distance between immune cytopenia onset and IEI identification was 5.4 years (-7.0-18.6 years) (Table 2).

Besides hematological presentation, we reported exclusively immunological parameters presenting with a significant difference between the two groups. The other variables analyzed were described in Supplementary table.

\section{Laboratory investigations}

The altered parameters of lymphocyte typing showing a predominance in IEI+ group were the following: lymphopenia ( $\mathrm{p}=0.009)$, a reduction below the lower limit of normal (LLN) in CD4+ nayive T-cells\% ( $\mathrm{p}=0.001)$, CD8+ nayive T-cells\% ( $\mathrm{p}=0.022)$, T-reg cells\% $(\mathrm{p}=0.009)$ and switched memory B-cells\% $(\mathrm{p}=0.008)$, an increase over the upper limit of normal in CD4+ effector memory T-cells\% $(\mathrm{p}=0.001)$ and CD21low B-cells\% $(\mathrm{p}=0.003)$ (Table 3$)$. There was a difference in mean lymphocyte count $(\mathrm{p}=0.001)$ between IEI+ and IEIgroups. Mean CD4+ T-cell count was inferior $(\mathrm{p}=0.017)$ in IEI+ group, the same goes for mean CD8+ Tcell count $(\mathrm{p}=0.004)$. There was a difference $(\mathrm{p}<0.001)$ in mean CD4+ nayive T-cell\% count between IEI+ and IEI- groups, as for CD8+ nayive T-cell\% count $(\mathrm{p}<0.001)$. Mean CD4+ central memory T-cell\% count was superior $(\mathrm{p}=0.002)$ in IEI+ group, the same goes for CD4+ effector memory T-cell\% count $(\mathrm{p}=0.001)$ and CD8+ effector memory T-cell\% count $(\mathrm{p}=0.002)$. CD19+ B-cell count was on average lower $(\mathrm{p}=0.002)$ in IEI+ group, as for CD19+ B-cell\% count $(\mathrm{p}=0.034)$ and CD21low B-cell\% count $(\mathrm{p}=0.009)$. There was a difference $(\mathrm{p}<0.001)$ in switched memory B-cell\% count between IEI+ and IEI- groups, the same goes for plasmablast\% count $(\mathrm{p}=0.036)$ (Table 4). In IEI+ group, CD4+ nayive T-cells\% showed a positive correlation with $\mathrm{CD} 8+$ nayive T-cells\% (Pearson's $\mathrm{R}=0.769$ ) and a negative correlation with CD4+ effector memory T-cells\% (Pearson's $\mathrm{R}=-0.660$ ) and CD21low B-cells\% (Pearson's R=-0.627). Furthermore, in CVID subgroup, we observed a negative correlation between CD4+ nayive T-cells\% and CD21low B-cells\% (Pearson's $\mathrm{R}=-0.453$ ) (Fig. 2).

Hypogammaglobulinemia involving at least one class among IgG, IgA, IgM was more frequent in IEI+ group ( $89 \%$ vs $36 \%, p=0.001)$, the same goes for hypogammaglobulinemia involving at least two classes (68\% vs $12 \%, \mathrm{p}<0.001)$ (Table 3$)$. Mean IgG count was inferior $(\mathrm{p}=0.002)$ in IEI+ group, as for $\operatorname{IgA}(\mathrm{p}=0.010)$ and $\operatorname{IgM}(\mathrm{p}<0.001)$ (Table 4).

Molecular analysis

In 9/47 patients, diagnosed as CVID, we performed genetic analysis as previously described: in $3 / 9$ patients we did not identified any pathogenic variants or variants of uncertain significance (VUS); in $6 / 9$ patients we found the variants summarized in Table 1-A.

Most of the variants are classified as VUS, with the exception of CR2:c.826delT classified as pathogenic, PRF1:c.272C >A and TNFRSF13B:c.260T $>$ A classified as likely pathogenic.

We found that $2 / 6$ patients carried TNFRSF13B variants coding for TACI receptor, showing respectively the monoallelic variant p.Ile87Asn and the compound heterozygous variants p.Ser144Ter and p.Cys193Arg. 
3/47 patients, diagnosed as ALPS Type IA, carried pathogenic variants in the TNFRSF6 gene, coding for FAS receptor (Table 1-B). The patient affected by DiGeorge syndrome showed a $2 \mathrm{Mb}$ deletion on chromosome 22 from $C L T C L-1$ gene to LRZTR1 gene.

\section{DISCUSSION}

This study found a strong association between immune cytopenias and IEI, demonstrating the feasibility and usefulness of an extended lymphocyte immunophenotyping to detect IEI signs in immune cytopenic pediatric patients. In this context, we identified $\mathrm{T}$ and $\mathrm{B}$ lymphopenia, a reduction in CD4+ and CD8+ nayive T-cells\%, T-reg cells\% and switched memory B-cells\%, and an increase in CD4+ effector memory Tcells\% and CD21low B-cells\% along with the presence of hypogammaglobulinemia as variables significantly associated with IEI.

Mean lymphocyte, CD4+ and CD8+ T-cell count reached lower mean levels in IEI+ group. Lymphopenia is indeed one of the factors of Immunodeficiency related score, a diagnostic algorithm developed for an early identification of IEI ${ }^{13}$; thus, it is a suggestive sign of $\mathrm{CID}^{11}$, described also in individuals with $\mathrm{CVID}^{14}$.

A general reduction in CD4+ T-cells probably led to a decline in T-reg cells, more prevalent in IEI+ group and responsible for the impairment of self-tolerance mechanism that frequently occurs in subjects with IEI ${ }^{15}$.

T-cell phenotype in IEI+ group showed a decrease in CD4+ and CD $8+$ nayive T-cells\% against an expansion in CD4+ and CD8+ effector memory T-cells\%, being common features of CVID ${ }^{16}$, reported also in DGS ${ }^{17}$, likely due to persistent immune system stimulation by chronic/recurrent infections. Diverging from literature, we found increased CD4+ central memory T-cells\% in IEI+ group, as a potential consequence of their abnormal interaction with antigen-presenting cells and subsequent failed differentiation into effector memory T-cells ${ }^{18}$.

B-cell phenotype was characterized in IEI+ group by an accumulation of CD21low B-cells\% against a decrease in PAN-B\%, switched memory B-cells\% and plasmablasts\%, whose lack resulted in a defective production of $\operatorname{IgG}$, IgA and IgM. This is in line with the fact that switched memory B-cells\%, IgG and IgA deficiency and CD21low B-cells\% expansion are diagnostic criteria of CVID $^{11}$, which is the most frequent IEI in literature ${ }^{19}$ as well as in our cohort.

In IEI+ group, CD4+ nayive T-cells\% showed a positive correlation with CD8+ nayive T-cells\% and a negative correlation with CD4+ effector memory T-cells\% and CD21low B-cells\%. The negative correlation between CD4+ nayive T-cells\% and CD21low B-cells\% maintained significance in our CVID subgroup, as reported by Warnatz ${ }^{20}$. All these associations probably reflect the role of persistent/chronic inflammationand infection-driven immune activation in the impairment of T-cell regulation of B-cells ${ }^{20}$, resulting in an immunosenescent-like phenotype typical of some IEI forms ${ }^{21}$. These features were also found in patients suffering simultaneously from both CVID and ITP $^{22}$, characterized by an accumulation of auto-reactive CD21low B lymphocytes, responsible for IEI-related autoimmune disorders ${ }^{8}$, along with defects in PAN-B cells bone marrow production and CD4+ nayive T-cells differentiation. These findings suit also with the individual of the cohort with DGS, showing low CD4+ nayive T-cells\%, switched memory B-cells\% and IgM levels, along with an inverted $\mathrm{CD} 4+/ \mathrm{CD} 8+$ ratio, all biomarkers configuring an immunophenotype associated with autoimmunity, lymphoproliferation and a severe clinical course, characterized in our case by invasive and recurrent infections ${ }^{23-25}$. Memory and transitional B-cells\% did not show a significant difference between the two groups. This result could be indicative of continuous autoantigen-driven stimulation and defective immune-regulatory networks, previously described in subjects with autoimmune manifestations, including ITP ${ }^{26,27}$, which was the most represented immune cytopenia in our two groups.

We identified a high rate of correlation between immune cytopenias and IEI, probably due to the inclusion criteria of the cohort, in which persistent/relapsing forms of immune cytopenias affected $97 \%$ of patients. Our hypothesis is supported by Hadjadj, assessing the pathogenic role and poor prognostic significance of proven and/or potential IEI-related genes in $65 \%$ of pediatric ES $\operatorname{cases}^{28}$.

We found two individuals with CVID carrying TNFRSF13B variants. The frequency of TNFRSF13B gene 
mutations was reported to be significantly higher in CVID compared to healthy controls and TACI biallelic mutations were detected only in CVID. Patients with biallelic TACI mutations had a similar incidence of autoimmunity and lymphoproliferation compared to wild-type TACI subjects. CVID individuals carrying monoallelic mutations had a severe clinical course with the highest prevalence of immune-dysregulation disorders $^{29}$. Interestingly, the Pt.2 patient carried also three other alterations, the pathogenic variants CR2:c.826delT and PRF1:A91V and the VUS TCF3:G444E (Table 1-A). Further studies are needed to validate these additional variants and to understand if they act in concert with biallelic genetic alterations in TNFRSF13B to give rise to complex IEI-related phenotype.

This study's primary limitations result from the sample size and the retrospective nature of the review. The high diagnosis rate of IEI in such a relatively limited cohort could be related to subjects' enrollment at a tertiary care center, where immunodeficiency suspicion may be elevated.

The strength of this study consists in an in-depth clinical/immunological characterization of the cohort highlighting correlations useful in the prompt identification and management of immune cytopenic children with higher risk of IEI.

In conclusion, IEI diagnosis in patients with immune cytopenias was significantly associated with specific clinical signs and immunophenotypical anomalies, namely T/B lymphopenia, decrease in nayive T-cells\%, switched memory B-cells\%, plasmablasts\% and immunoglobulins, increase in effector/central memory Tcells\% and CD21low B-cells\%. Thus, an extended lymphocyte typization can identify subjects worthy of IEI-related molecular analysis, useful for validating new targeted-gene variants and unveiling their correlation with IEI phenotype, which in some cases is the expression of a complex genotypic interaction between a small number of mutant genes rather than of a single-gene inherited Mendelian disorder.

Authorship Contributions Drs Zama, Conti, Moratti and Cantarini conceptualized and designed the study, drafted the initial manuscript, and reviewed and revised the manuscript.

Dr Rivalta designed the data collection instruments, collected data and critically revised the manuscript.

Dr Rondelli carried out statistical analyses and critically revised the manuscript.

Drs Facchini, Prete and Ferrari contributed to interpretation of data and critically revised the manuscript.

Prof Seri carried out molecular analyses and critically revised the manuscript.

Prof Pession critically reviewed the manuscript for important intellectual content and contributed to the design of the study.

All authors approved the final manuscript as submitted and agree to be accountable for all aspects of the work.

\section{REFERENCES}

1. Ghaithi IA, Wright NAM, Breakey VR, et al. Combined Autoimmune Cytopenias Presenting in Childhood. Pediatr Blood Cancer . 2016;63(2):292-298. doi:10.1002/pbc.25769

2. Aladjidi N, Leverger $\mathrm{G}$, Leblanc $\mathrm{T}$, et al. New insights into childhood autoimmune hemolytic anemia: a French national observational study of 265 children. Haematologica . 2011;96(5):655-663. doi:10.3324/haematol.2010.036053

3. Aladjidi N, Fernandes H, Leblanc T, et al. Evans Syndrome in Children: Long-Term Outcome in a Prospective French National Observational Cohort. Front Pediatr . 2015;3:79. doi:10.3389/fped.2015.00079

4. Rivalta B, Zama D, Pancaldi G, et al. Evans Syndrome in Childhood: Long Term Follow-Up and the Evolution in Primary Immunodeficiency or Rheumatological Disease. Front Pediatr . 2019;7:304. doi:10.3389/fped.2019.00304 
5. Seidel MG. Autoimmune and other cytopenias in primary immunodeficiencies: pathomechanisms, novel differential diagnoses, and treatment. Blood . 2014;124(15):2337-2344. doi:10.1182/blood-2014-06-583260

6. Rotz SJ, Ware RE, Kumar A. Diagnosis and management of chronic and refractory immune cytopenias in children, adolescents, and young adults.Pediatr Blood Cancer . 2018;65(10). doi:10.1002/pbc.27260

7. Fischer A, Provot J, Jais J-P, et al. Autoimmune and inflammatory manifestations occur frequently in patients with primary immunodeficiencies. J Allergy Clin Immunol . 2017;140(5):1388-1393.e8. doi:10.1016/j.jaci.2016.12.978

8. Rakhmanov M, Keller B, Gutenberger S, et al. Circulating CD21low B cells in common variable immunodeficiency resemble tissue homing, innate-like B cells. Proc Natl Acad Sci U S A . 2009;106(32):13451-13456. doi:10.1073/pnas.0901984106

9. Lucas CL, Kuehn HS, Zhao F, et al. Dominant-Activating, Germline Mutations in Phosphoinositide 3-Kinase p110ס Cause T Cell Senescence and Human Immunodeficiency. Nat Immunol . 2014;15(1):88-97. doi:10.1038/ni.2771

10. Tesi B, Davidsson J, Voss M, et al. Gain-of-function SAMD9L mutations cause a syndrome of cytopenia, immunodeficiency, MDS, and neurological symptoms. Blood . 2017;129(16):2266. doi:10.1182/blood-201610-743302

11. Seidel MG, Kindle G, Gathmann B, et al. The European Society for Immunodeficiencies (ESID) Registry Working Definitions for the Clinical Diagnosis of Inborn Errors of Immunity. J Allergy Clin Immunol Pract . 2019;7(6):1763-1770. doi:10.1016/j.jaip.2019.02.004

12. Walter JE, Ayala IA, Milojevic D. Autoimmunity as a continuum in primary immunodeficiency. Curr Opin Pediatr . 2019;31(6):851-862. doi:10.1097/MOP.0000000000000833

13. Yarmohammadi H, Estrella L, Doucette J, Cunningham-Rundles C. Recognizing primary immune deficiency in clinical practice. Clin Vaccine Immunol CVI . 2006;13(3):329-332. doi:10.1128/CVI.13.3.329332.2006

14. Wong GK, Huissoon AP. T-cell abnormalities in common variable immunodeficiency: the hidden defect. J Clin Pathol . 2016;69(8):672-676. doi:10.1136/jclinpath-2015-203351

15. Grimbacher B, Warnatz K, Yong PFK, Korganow A-S, Peter H-H. The crossroads of autoimmunity and immunodeficiency: Lessons from polygenic traits and monogenic defects. J Allergy Clin Immunol . 2016;137(1):3-17. doi:10.1016/j.jaci.2015.11.004

16. Nechvatalova J, Pavlik T, Litzman J, Vlkova M. Terminally differentiated memory T cells are increased in patients with common variable immunodeficiency and selective IgA deficiency. Cent-Eur J Immunol . 2017;42(3):244-251. doi:10.5114/ceji.2017.70966

17. Marcovecchio GE, Bortolomai I, Ferrua F, et al. Thymic Epithelium Abnormalities in DiGeorge and Down Syndrome Patients Contribute to Dysregulation in T Cell Development. Front Immunol . 2019;10. doi:10.3389/fimmu.2019.00447

18. Pepper M, Jenkins MK. Origins of CD4(+) effector and central memory T cells. Nat Immunol . 2011;12(6):467-471. doi:10.1038/ni.2038

19. Modell V, Knaus M, Modell F, Roifman C, Orange J, Notarangelo LD. Global overview of primary immunodeficiencies: a report from Jeffrey Modell Centers worldwide focused on diagnosis, treatment, and discovery.Immunol Res . 2014;60(1):132-144. doi:10.1007/s12026-014-8498-z

20. Warnatz K, Schlesier M. Flowcytometric phenotyping of common variable immunodeficiency. Cytometry B Clin Cytom . 2008;74B(5):261-271. doi:10.1002/cyto.b.20432 
21. Cannons JL, Preite S, Kapnick SM, Uzel G, Schwartzberg PL. Genetic Defects in Phosphoinositide 3-Kinase $\delta$ Influence CD8(+) T Cell Survival, Differentiation, and Function. Front Immunol . 2018;9:17581758. doi:10.3389/fimmu.2018.01758

22. Stuchlý J, Kanderová V, Vlková M, et al. Common Variable Immunodeficiency patients with a phenotypic profile of immunosenescence present with thrombocytopenia. Sci Rep . 2017;7:39710.

23. Nain E, Kiykim A, Ogulur I, et al. Immune system defects in DiGeorge syndrome and association with clinical course. Scand J Immunol . 2019;90(5):e12809. doi:10.1111/sji.12809

24. Montin D, Marolda A, Licciardi F, et al. Immunophenotype Anomalies Predict the Development of Autoimmune Cytopenia in 22q11.2 Deletion Syndrome. J Allergy Clin Immunol Pract . 2019;7(7):2369-2376. doi:10.1016/j.jaip.2019.03.014

25. Sullivan KE. Immune Biomarkers of Autoimmunity in Chromosome 22q11.2 Deletion Syndrome. J Allergy Clin Immunol Pract . 2019;7(7):2377-2378. doi:10.1016/j.jaip.2019.04.051

26. Li X, Zhong H, Bao W, et al. Defective regulatory B-cell compartment in patients with immune thrombocytopenia. Blood . 2012;120(16):3318-3325. doi:10.1182/blood-2012-05-432575

27. Zahran AM, Aly SS, Elabd A, Mohamad IL, Elsayh KI. Regulatory and memory B lymphocytes in children with newly diagnosed immune thrombocytopenia. J Hematol . 2017;6(4):81-86-86. doi:10.14740/

28. Hadjadj J, Aladjidi N, Fernandes H, et al. Pediatric Evans syndrome is associated with a high frequency of potentially damaging variants in immune genes. Blood . Published online January 1, 2019:blood-2018-11887141. doi:10.1182/blood-2018-11-887141

29. Pulvirenti F, Zuntini R, Milito C, et al. Clinical Associations of Biallelic and Monoallelic TNFRSF13B Variants in Italian Primary Antibody Deficiency Syndromes. J Immunol Res . 2016;2016. doi: $10.1155 / 2016 / 8390356$

Tables

Table 1 IEI-related molecular analysis through a specific IEI targeted gene panel

Abbreviations: ALPS, Autoimmune lymphoproliferative syndrome; CVID, Common variable immune deficiency; VUS, Variants of uncertain significance.

Table 2 General characterization of cohort, IEI+ and IEI- groups (SD: standard deviation)

Abbreviations: ALPS, Autoimmune lymphoproliferative syndrome; CI, confidence interval; CVID, Common variable immune deficiency; IEI, Inborn errors of immunity; y, years. + Data by definition not regarding IEI- group. ++ No statistical analysis conducted on the data.

Table 3 Extended lymphocyte typization comparison between IEI+ and IEI- groups (qualitative variables)

Abbreviations: IEI, Inborn errors of immunity; obs, observations.

Legend: -, decrease; - increase.+ \% total lymphocytes.++ \% total CD $4+$ cells. SS \% total CD8+ cells. $\Pi \% T^{" P} \mathrm{~Pa} \beta+{ }^{\prime \prime} \Delta 3+\varsigma \in \lambda \lambda \varsigma$. $++\%$ total CD19+ cells. ++++ Only IgG, IgA and IgM classes were analysed.

Table 4 Extended lymphocyte typization comparison between IEI+ and IEI- groups (quantitative variables) (SD: standard deviation)

Abbreviations: IEI, Inborn errors of immunity; obs, observations; WBC, white blood cells. + \% total lymphocytes. $++\%$ total CD $4+$ cells. $\S \%$ total CD $8+$ cells. $1 \%$ TCR $\alpha \beta+$ CD3 + cells. $++\%$ total CD19+ cells ++++ SI conversion factor: To convert IgG/IgA/IgM to g/L, multiply values by $10^{2}$. ++++ SI conversion factor: To convert IgG/IgA/IgM to g/L, multiply values by 102 .

\section{Supplementary table}


Comparison of qualitative clinical, laboratory and therapeutic variables between IEI+ and

IEI- groups

Abbreviations: DTP, Diphtheria-Tetanus-Pertussis; IEI, Inborn errors of immunity; IVIG, intravenous immunoglobulins; MMR, Measles-Mumps-Rubella; obs, observations. Legend: —, decrease; -, increase.+ Familial immune-mediated disorders (endocrinological, gastrointestinal, hematological, mucocutaneous, pulmonary and rheumatological). ++ Invasive infections (bronchopneumonia, cutaneous abscesses, osteomyelitis, diffuse and/or profound herpetic lesions).SS Recurrent/relapsing infections (recurrent urinary and respiratory tract infections, mycosis/parasitosis relapsing at least one time). P Mucocutaneous disorders (alopecia, aphthosis, dermatitis, granulomas, melanocytic nevi, rosacea, urticarial vasculitis and warts). ++ Pulmonary disorders (asthma, bronchiectasis, granulomatous-lymphocytic interstitial lung disease, other interstitiopathies). ++++ Allergies (asthma, dermatitis, drug and food allergies/intolerances, rhinoconjunctivitis, Vernal keratoconjunctivitis).SSSSAntibody responses to MMR and DTP vaccination detected respectively through chemiluminescent (microparticle) immunoassay and enzyme immunoassay.

\section{Figure legends}

Fig. 1 Immune cytopenias and IEI-related forms: prevalence assessment in a pediatric cohort

Abbreviations: AIHA, Autoimmune hemolytic anemia AIN, Autoimmune neutropenia; ITP, Immune thrombocytopenia; IEI, Inborn errors of immunity; pts, patients.

Fig. 2 Significant correlations between lymphocyte subsets in IEI+ group and CVID subgroup

Abbreviations: CVID, Common variable immune deficiency; IEI, Inborn errors of immunity.Legend: - , decrease; - increase. (A) Scatter plot describing the correlation existing between CD $4+$ naive $T$ cells (\%) (X-axis) and CD8+ naive $T$ cells (\%) (Y-axis) in IEI+ group through Pearson's correlation coefficient. (B) Scatter plot describing the correlation existing between $C D_{4}+$ naive $T$ cells (\%) (X-axis) and CD $4+$ effector memory T cells (\%) (Y-axis) in IEI+ group through Pearson's correlation coefficient.(C) Scatter plot describing the correlation existing between CD4+ naive T cells (\%) (X-axis) and CD21low B cells (\%) (Y-axis) in IEI+ group through Pearson's correlation coefficient.(D) Scatter plot describing the correlation existing between CD4+ naive T cells (\%) (X-axis) and CD21low B cells (\%) (Y-axis) in CVID subgroup through Pearson's correlation coefficient.

\section{Hosted file}

Table 1.pdf available at https://authorea.com/users/372520/articles/490522-immunophenotypeidentifies-children-with-immune-cytopenias-at-risk-of-inborn-errors-of-immunity-relatedmutations

\section{Hosted file}

Table 2.pdf available at https://authorea.com/users/372520/articles/490522-immunophenotypeidentifies-children-with-immune-cytopenias-at-risk-of-inborn-errors-of-immunity-relatedmutations

\section{Hosted file}

Table 3.pdf available at https://authorea.com/users/372520/articles/490522-immunophenotypeidentifies-children-with-immune-cytopenias-at-risk-of-inborn-errors-of-immunity-relatedmutations

\section{Hosted file}

Table 4.pdf available at https://authorea.com/users/372520/articles/490522-immunophenotypeidentifies-children-with-immune-cytopenias-at-risk-of-inborn-errors-of-immunity-relatedmutations 


\section{Hosted file}

Figure 1.pdf available at https://authorea.com/users/372520/articles/490522-immunophenotypeidentifies-children-with-immune-cytopenias-at-risk-of-inborn-errors-of-immunity-relatedmutations

\section{Hosted file}

FIGURE 2.pptx available at https://authorea.com/users/372520/articles/490522-immunophenotypeidentifies-children-with-immune-cytopenias-at-risk-of-inborn-errors-of-immunity-relatedmutations 